\title{
Blogchain - Disruptives Publizieren auf der Blockchain
}

\author{
Clemens H. Cap D Benjamin Leiding
}

Eingegangen: 1. Juli 2018 / Angenommen: 28. September 2018 / Online publiziert: 11. Oktober 2018 (C) Der/die Autor(en) 2018

Zusammenfassung Wir stellen ein neues Konzept als Metamodell für das wissenschaftliche Publikationswesen vor. Unser Konzept ist im Kontext eines dreistufigen Phasenmodells digitaler Disruption von Geschäftsprozessen angesiedelt. Die erste Phase besteht dabei aus Technologie ohne Prozessanpassung. Die zweite Phase umfasst eine Prozessanpassung unter der Kontrolle von Intermediären und führt zu unerwünschter aber schwer vermeidbarer Zentralisierung. Die dritte Phase durchbricht schließlich die Vormachtstellung intermediärer Institutionen und nutzt dazu die disruptiven Möglichkeiten der Blockchain-Technologie.

Die Anwendung dieser Technologie erlaubt eine Veränderung der Geschäftsprozesse bestehender Zeitschriften, macht die Rolle des Verlags als Intermediär überflüssig und verspricht eine Lösung des Problems der Kostenexplosion in der wissenschaftlichen Literaturversorgung. Wir stellen Ergebnisse einer theoretischen Machbarkeitsstudie vor und präsentieren eine erste Implementierung als Proof-of-Concept. Diese werden als Basis für ein Feldexperiment dienen.

Schlüsselwörter Digitales Publizieren · Geschäftsmodelle für die Blockchain · Anwendungen der Blockchain-Technologie

C. H. Cap $(\bowtie)$

Lehrstuhl für Informations- und Kommunikationsdienste, Institut für Informatik, Universität Rostock, Rostock, Deutschland

E-Mail: clemens.cap@uni-rostock.de

B. Leiding

Lehrstuhl für Telematik, Institut für Informatik, Universität Göttingen, Göttingen, Deutschland

E-Mail: benjamin.leiding@informatik.uni-goettingen.de 


\title{
Blogchain - Disruptive Publishing on the Blockchain
}

\begin{abstract}
We present a metamodel and a new concept for scientific publishing. Our concept is positioned in the context of a three phase model of digital disruption for business processes. The first phase consists of technology without process adaptation. In the second phase, processes are modified under the control of intermediaries. This leads to undesired centralisation, which is difficult to avoid. The third phase breaks the dominance of intermediary institutions and uses the disruptive properties of blockchain technology.

Applying blockchain technology, business processes of existing journals can be modified and renders superfluous the role of a publishing house as an intermediary. It promises a solution for the cost explosion in scientific journals. We present results of a theoretical analysis and report on a first implementation as proof-of-concept. Both shall form the starting point for a field experiment.
\end{abstract}

Keywords Digital publishing - Business models for the blockchain - Applications of blockchain technology $\cdot$ Blockchain publishing

\section{Einleitung}

Das Publikationssystem befindet sich in einer Krise. Man kann die Gründe ganz abstrakt in soziologischer Kritik am Wissenschaftssystem suchen. So schildern Stölting und Schimank (2001) den Streit zwischen der Bewahrung etablierter, bewährter Strukturen und dem Druck, die Trägheit von Entscheidungsmechanismen und Personalstrukturen zu durchbrechen. Man kann auch die weithin geführte Diskussion über die Replikationskrise aufgreifen. Bestehen Zusammenhänge zwischen einem immer weiter wachsenden, primär quantitativen Publikationsdruck und einer Bevorzugung spektakulär vermarktbarer Resultate? Vergessen wir die in vielen Bereichen so wichtige Wiederholung von Experimenten, wenn diese nicht kurzfristig und sicher Veröffentlichungen in Zeitschriften mit hohem impact factor versprechen? Das Problem erscheint dringend und die DFG denkt über Zusammenhänge mit quantitativ parametrisierenden Steuerungs-, Bewertungs- und Gratifikationssystemen nach (Hartig 2017).

Das Publikationswesen ist ferner durch ökonomische Verwerfungen und finanzielle Auseinandersetzungen beachtlicher Größenordnung geprägt. Auf der einen Seite sind die Kosten im wissenschaftlichen Verlagswesen durch die Digitalisierung in den letzten 10 Jahren dramatisch gesunken. Die Aufgaben des Editierens und Setzens werden im Zeitalter von Word und LaTeX durch die Autoren selber übernommen, die Begutachtung erfolgt durch Personen, die durch ihre Position im Wissenschaftssystem bereits finanziert sind und die Koordination wird durch die Workflow-Komponenten automatisierter Einreichsysteme erledigt. Die verbleibenden Kosten der Fertigung und Versendung sind durch das Internet de facto auf null gefallen (siehe Infobox 1). 


\section{Infobox 1}

Kosten wissenschaftlicher Publikation - eine Überschlagsrechnung

- Pro Terabyte und Monat langsamen Archivspeicher: 4 US-\$

- Pro Terabyte und Monat Plattenspeicher: Zwischen 25 und 45 US-\$

- Pro Terabyte Datentransfer: Zwischen 2 und 20 US-\$

Die Datenmenge aller in der US Library of Congress in Printform gelagerten Publikationen beträgt nach Schätzungen im Internet zwischen 15 und 5000 Terabyte, wobei über die Zählweise gestritten werden kann (Johnston 2012). Wesentlich ist uns hier die Veranschaulichung der Größenordnung. Wenige durchschnittliche Monatseinkommen eines europäischen Haushalts reichen aus, um sämtliche Wissensbestände der weltweit größten Bibliothek einen Monat lang zu speichern oder einmal vollständig durch das Internet zu übertragen

Auf der anderen Seite sind die Preise für den Leser in astronomische Höhen gestiegen. Diese Preiserhöhungen lassen sich ökonomisch nicht rechtfertigen. So befinden sich die Umsatzrenditen von Wissenschaftsverlagen in der Größenordnung von 30-40\% $\%^{1}$, während im Vergleich die deutsche Metall- und Elektroindustrie fast durchwegs unter $4 \%$ bleibt $^{2}$, die Baubranche bei $6 \%$ und der Handel bei $3 \%{ }^{3}$.

Die Einführung des Open Access Konzepts hat zwar zunächst grundlegende Impulse versprochen, jedoch keine Lösung gebracht. Jeder Wissenschaftler kennt die dramatische Zunahme von predatory publishers, bei denen neu gegründete und oftmals dubiose Institutionen die Autoren verfolgen um sie zu einer Einreichung überarbeiteter Papiere zu bemühen, deren Begutachtungssystem wenig selektiert und deren Geschäftsmodell sich auf open access publication charges stützt. Zugleich aber haben diese Zahlungsströme eine beachtenswerte und selten durch die Dienstleistung gerechtfertigte Höhe (siehe Infobox 2).

Digitalisierung hat bekanntlich bei einer ganzen Reihe von Branchen zu massiven disruptiven Veränderungen geführt. Wir werden in Abschn. 2 ein mögliches strukturelles Modell für solche Veränderungen vorstellen und argumentieren, weshalb gerade die Blockchain-Technologie ein wesentlicher Treiber der nächsten Phase der Disruption nach diesem Modell sein kann. Anschließend arbeiten wir in Abschn. 3 heraus, welche Aspekte bei wissenschaftlichen Publikationen wesensprägend und daher unverzichtbar sind und welche sich allein durch die ökonomischen Zwänge sich verändernder Technologien begründen. In Abschn. 4 stellen wir die „Blogchain“ als jenes Modell wissenschaftlichen Publizierens vor, das sich aus dieser Anforderungsanalyse in Abschn. 3 unter Nutzung der Blockchain als Technologie ergibt.

\footnotetext{
1 https://www.timeshighereducation.com/blog/it-time-nationalise-academic-publishers und https://oa2020de.org/blog/2018/03/06/oa-statt-verstaatlichung-Wissenschaftsverlage/.

2 https://de.statista.com/statistik/daten/studie/153677/umfrage/ertragslage-der-metall--und-elektroindustriein-deutschland-seit-1997/.

3 https://www.kfw.de/PDF/Download-Center/Konzernthemen/Research/PDF-Dokumente-KfW-Mittel standspanel/KfW-Mittelstandspanel-2016.pdf.
} 
Wir schließen mit einer kritischen Analyse, einer Vorstellung aktueller Umsetzungsversuche und einem Vergleich mit ähnlichen Ansätzen.

Infobox 2 Nach Untersuchungen der Universität Bielefeld beträgt der Median an Open Access Publication Charges bei deutschen Universitäten über 50.000 Publikationen gerechnet $1759 €$, wobei es Ausreißer bis hin zu 25.000€ gibt. Damit kann der Meinung entgegengetreten werden, dass das Open Access Prinzip bereits eine Lösung der hier beschriebenen Publikationskrise und der entsprechenden ökonomischen Verzerrungen darstellt.

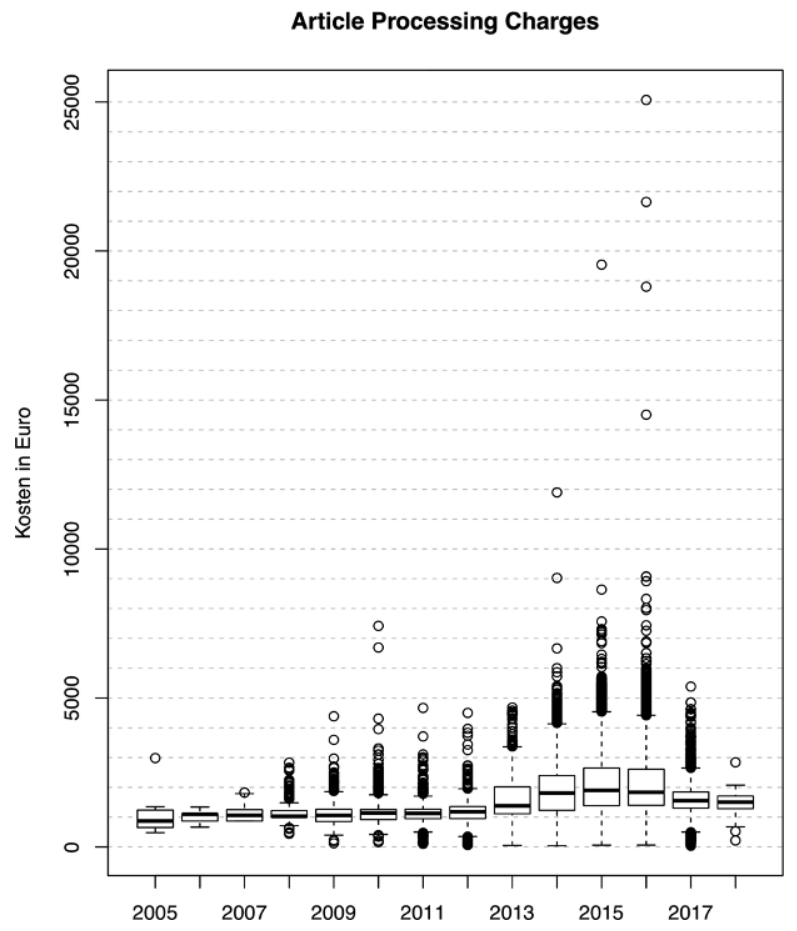

\section{Die 3 Phasen digitaler Disruption}

Digitalisierung hat zu einer Veränderung vieler Geschäftsabläufe geführt. Wir stellen diese in ein Modell aus drei Phasen, um die jeweiligen Treiber der Entwicklung zu illustrieren. Ziel ist dabei nicht eine Beschreibung, welche die geschehenen Veränderungen in Branchen im Nachhinein analysiert und methodisch durch empirische Auswertung validiert; Ziel ist jedoch eine Modellierung, welche die gerade geschehenden Veränderungen zu verstehen versucht, um ihnen für die Zukunft eine Richtung und visionäre Gestaltungskraft zu verleihen. Unser Narrativ der drei Phasen stellt dabei nur eine mögliche Sichtweise auf digitale Disruption dar. Andere 
Perspektiven sind denkbar, doch die gewählte Form eignet sich besonders gut zur Erläuterung der Motivation unserer Idee.

\subsection{Phase 1: Erkenntnis-Phase}

Phase 1, die wir als Erkenntnis-Phase bezeichnen wollen, ist von der Beobachtung geprägt, dass die neuen technischen Möglichkeiten der Informations- und Kommunikations-Technologie bestimmte, uns wohlbekannte Prozesse beschleunigen und effizienter machen können. In der Folge werden diese Beschleunigungen auch eingesetzt.

In der Kommunikation wird beispielsweise Email genutzt und im Unterricht die Verteilung von Lehrmaterialien über PDF. Die neueren Unterstützungsformen der Technologie sind aber noch nicht bekannt, noch nicht implementiert oder noch nicht gesellschaftlich etabliert. Die über Email weit hinausgehenden Metaphern von Lebenslinien oder Status-Meldungen, wie wir sie heute von Facebook oder Twitter her kennen, sind noch nicht erfunden, auch die vielfältigen digitalen Lehrformen sind noch nicht angedacht. Das Ende der Phase 1 wird eingeläutet, sobald die technologische Unterstützung bisheriger Geschäftsprozesse so gut erfolgt, dass deren Grenzen deutlich werden.

\subsection{Phase 2: Prozessbildungs-Phase}

In Phase 2, die wir Prozessbildungs-Phase oder Phase der Intermediäre nennen, verändern Ideengeber die bestehenden Abläufe so, dass sie besser mit der neuen Technologie zusammenspielen.

Wir betrachten als Beispiel wieder die Kommunikation von Personen mit anderen Personen, Gruppen und Unternehmen. Die dem Brief nachgebildete Email wird durch neue Metaphern ergänzt, die keine Entsprechung oder Vorbilder in klassischen Technologien haben. Es entstehen soziale Netze (wie Facebook, Google+), Kurznachrichten- und Status-Dienste (wie Twitter), Kooperationsplattformen (wie GoogleDocs, Etherpad, Overleaf) und andere. Diese neuen Formen spezialisieren sich schließlich immer weiter in Portale, welche die Prozesse ganz bestimmter Lebenslagen und Bedürfnisse analysiert haben und optimal unterstützen wollen. Charakteristische Beispiele sind hier Uber, AirBnB, Tinder, Slack oder Github.

Die entstehenden Lösungen skalieren und werden weltweit angeboten. Da sie grundsätzlich leicht zu kopieren sind, behaupten sie sich gegen Konkurrenz meistens durch Lock-In-artige Mechanismen und ihre Monopolstellung, die sich aus der Rolle als enabling intermediaries ergibt. Netzwerk-ökonomisch machen mehrere Facebooks, Ubers, AirBnBs oder Tinders auch wenig Sinn, denn das Ziel ist die one stop platform auf der sich eben alle Freunde, Fahrgelegenheiten, Übernachtungsmöglichkeiten oder Flirtpartner kontaktieren lassen.

In den meisten Branchen befinden wir uns derzeit in der Phase 2 und entdecken gerade die dieser Phase innewohnenden Probleme: Lösen die Intermediäre ihre Aufgabe gut, so generieren sie aufgrund ihrer Machtposition rasch enorme Profite, ganz ähnlich wie die Wissenschaftsverlage. Sie dominieren den Markt durch ihre Kenntnis unserer Daten, Profile und Präferenzen und verursachen damit Fragen nach 
Datenschutz und Privatheit. Sie verwenden diese Kenntnis zur weiteren Steigerung eigener Profite und können sich dabei dieser Daten bedienen. Die aktuellen Debatten über den Missbrauch von Persönlichkeitsprofilen auf Facebook durch die Firma Cambridge Analytica und die möglichen Folgen für den amerikanischen Wahlausgang 2016 finden sich derzeit in den Schlagzeilen.

Für die später folgende Anwendung dieses Modells ist es wichtig, von der jeweiligen Rolle der Intermediäre etwas zu abstrahieren, denn die genauere Bedeutung variiert hier sehr stark. So sind die Funktionen als content gatekeeper in sozialen Netzen, bei Zwischenhändlern und Vermittlungsplattformen (wie Uber und AirBnB) und bei Wissenschaftsverlagen bei genauerer Betrachtung unterschiedlich. Insbesondere tritt bei Verlagen zusätzlich noch die Funktion der Vermittlung von Begutachtungen hinzu.

\subsection{Brauchen wir Intermediäre?}

Die Frage liegt daher nahe, ob die gesellschaftliche Machtposition der Intermediäre gerechtfertigt ist, ob sie reguliert werden kann und muss, und ob andere, dezentrale Organisationsformen ihre Aufgabe übernehmen können.

Technisch ist die Frage seit langem positiv beantwortet. Friendica, Diaspora, Identica, Libertree, Mastodon, Movim, Twister und Galaxy2 sind nur einige der dezentralen digitalen sozialen Netze, die als Alternative zur Nutzung bereitstehen. Der geringe Bekanntheitsgrad und die noch geringere Verwendung dieser Systeme lässt Nachteile oder Probleme vermuten. Hier können insbesondere zwei Bereiche genannt werden: Wertschöpfung und Vertrauen.

Das Problem Wertschöpfung bedeutet, dass es diesen dezentralen Systemen an Anreizen für die skalierbare Bereitstellung der Dienstleistung in hohem Komfortgrad mangelt. Betrieb, Beratung, Fehlersuche und Weiterentwicklung der meisten dieser Systeme geschieht ohne stabile Geldflüsse und reduziert sich daher oft auf freiwillige Beiträge von Enthusiasten. Damit fehlen die Mittel für eine Bekanntmachung, für das Marketing und Branding, für systematische Benutzerstudien, für das Übersetzen in viele Fremdsprachen und etliche andere Formen des Un-Nerding, damit diese Dienste den Mainstream erreichen.

Das Problem Vertrauen bedeutet, dass es diesen Systemen an stabilen Mechanismen zum Aufstellen und Einhalten von Community-Standards mangelt. Das Problem ist vielschichtig und tiefliegend, es wurde aus mehreren Blickwinkel bereits erfolglos in Angriff genommen. Wir beschreiben einige Ansätze.

Aus algorithmischer Perspektive benötigt das Aufstellen und Einhalten von Community-Standards die Programmierung verteilter Konsensus-Algorithmen, um im Konfliktfall die unterschiedlichen Sichtweisen der einzelnen Teilnehmer einer gemeinsamen Abstimmung zuzuführen. Solche Verfahren sind in der Informatik zwar bekannt, sie führen aber auf verschiedene Probleme (Shen et al. 2009). Viele Ansätze haben quadratische Kommunikations-Komplexität und sind daher für größere Knotenzahlen nicht praktikabel. Zusätzlich lassen sich nach dem Brewerschen CAP-Theorem von den drei wünschenswerten Anforderungen CAP $(\mathrm{C}=$ Konsistenz, 
$A=$ Verfügbarkeit, $P=$ Überstehen von Netz-Partitionen) maximal zwei realisieren (Brewer 2000), (Gilbert und Lynch 2002). Eine technische Lösung scheint also vorerst - zu entfallen.

Die fehlende technische Lösung könnte durch organisatorische Maßnahmen gewährleistet werden. In der politischen Theorie wie in der Praxis mancher InformatikGroßprojekte ist das Modell des benevolent dictator bekannt, einer wohlwollenden aber mit diktatorischen Vollmachten ausgestatteten Integrationsfigur, welche den erwünschten Konsens im Interesse aller herbeiführt. Das Konzept scheint in manchen Bereichen erfolgreich, wie man etwa am Beispiel von Linus Torvalds beim LinuxProjekt sehen kann. Es ist aber fraglich, ob der Ansatz generell funktionieren kann, wie etwa die Geschehnisse um Cambridge Analytica und die bisherigen Reaktionen von Facebook und Marc Zuckerberg dazu zeigen.

\subsection{Phase 3: Demokratisierungs-Phase}

Die Blockchain-Technologie hat mit ihrer ersten Anwendung, dem Bitcoin, eindrücklich bewiesen, dass sie beide Problembereiche praktisch lösen kann. Auch wenn in der noch sehr jungen Technologie der crypto currencies viele offene Probleme bestehen, so können zwei Beobachtungen festgehalten werden, die Lösungen für die Probleme aus Abschn. 2.3 anbieten. Die Blockchain erlaubt eine dezentrale Verrechnung entstandener Wertschöpfung in Form von Crypto-Token; diese können gänzlich ohne zentrale Instanzen oder Intermediäre gebildet und direkt, Peerto-Peer ausgetauscht werden. Das Aufstellen und Einhalten von Community-Standards geschieht durch die Mehrheit der Betreiber von Blockchain-Knoten. Diese Mehrheit wird dabei zuverlässig und manipulationssicher gebildet und gewichtet, wobei die jeweiligen Blockchain Varianten nach Hashleistung (proof-of-work), Einsatz (proof-of-stake) oder externer Autorisierung (proof-of-authority, private Blockchain) unterscheiden ${ }^{4}$. Für die praktische Gewähr, dass die Blockchain-Technologie diese beiden Probleme tatsächlich stabil löst, ist kann als empirisches Argument die Marktkapitalisierung der Kryptowährungen von über $250 \mathrm{Mrd}$. $€$ genannt werden ${ }^{5}$, ist sie doch eine relativ hohe bounty oder ein hoher stake für ihre Fähigkeiten.

Wir stehen derzeit jedoch noch am Beginn dieser Entwicklung. Der Bitcoin zeigt mit über 1500 anderen Währungen, wie man sich ein dezentrales Währungssystem ohne Notenbanken vorstellen könnte. Man mag das Konzept einer Welt belächeln, in dem die Nationalbanken durch Millionen dezentraler Mining-Knoten ersetzt werden, doch es gibt einen wichtigen Fingerzeig, wie spannende Entwicklungen aussehen könnten. Auf ähnliche Weise könnte man von einem dezentralen Uber, Tinder oder AirBnB träumen: Eine dezentrale Suchmaschine schwingt sich nach Peer-to-PeerArt von Knoten zu Knoten und findet Partner für Autofahrten, Flirts oder Übernachtungen. Die Regeln des Handelns, die sich die Teilnehmer und Communities in freier Übereinkunft und ohne regulierende Intermediäre mit Eigeninteressen sel-

\footnotetext{
4 Vergleiche https://hackernoon.com/beginners-guide-what-s-the-difference-between-proof-of-workproof-of-stake-proof-of-burn-and-74c42df591ca.

5 Das ist der Wert, der nach https://coinmarketcap.com/all/views/all/ allen dort erfassten Kryptowährungen auf dem Markt nach Stand Juni 2018 größenordnungsmäßig zukommt.
} 
ber geben, werden durch Smart Contracts beschrieben und durch die Blockchain gewährleistet. Es ist zwar völlig unklar, welchen Anklang diese Nerd-Träume von einer Blockchain-Gesellschaft in unserer Gesellschaft haben werden, die technologischen Bausteine für ihre Umsetzung stehen jedoch bereit.

In die Demokratisierungs-Phase sind grundsätzlich auch andere Peer-to-Peer Technologien einzuordnen. Diese führen aber meist zu praktischen Problemen. Zu nennen sind beispielsweise unfaire Nutzungsformen (etwa das sogenannte free loading), die Übernutzung geteilter Ressourcen (also die tragedy of the commons) oder Sybil-Attacken. Letztere führen durch die massive Erstellung gefälschter digitaler Identitäten zu einem nicht repräsentativen Bild von der Gemeinschaft. Die Blockchain-Technologie nimmt derzeit eine Sonderstellung ein, da sie viele dieser Probleme zu lösen verspricht und das bei Kryptowährungen praktisch erfolgreich unter Beweis stellt.

\section{Wertschöpfung des wissenschaftlichen Publikationswesens}

Das wissenschaftliche Publikationswesen und der damit verbundene Prozess der Begutachtung soll aus der Vielzahl neuer Ideen jene herausschälen helfen, die sich dann langfristig gesellschaftlich als nützlich und hilfreich erweisen.

Die wissenschaftliche Methodik ist maßgeblich durch die Aufstellung von Gedankengebäuden (Thesen) geprägt, die anschließend einer kritischen Bewertung unterworfen werden. Es hängt nun von der spezifischen Disziplin ab, ob diese Bewertung durch ein Experiment oder eine Beobachtung erfolgt (Naturwissenschaften), durch kritisches Nachdenken (Logik, Mathematik, Philosophie), durch Umfragen oder Statistiken (Sozial-, Politik- und Wirtschaftswissenschaften), durch gesellschaftlich wahrgenommene Nützlichkeit (Ingenieurwissenschaften) oder durch komplexe Kombinationen (wie etwa in der Medizin oder in manchen Geistes- und Kulturwissenschaften). Allen Wissenschaften gemeinsam scheint das Bedürfnis nach Diskurs und gelegentlich auch Konsens der beteiligten Fachleute zu sein; dabei erzeugt der Wunsch nach Konsens einerseits und das Suchen nach neuen, originären Ideen ein bemerkenswertes Spannungsfeld. Ein weiterer Konfliktbereich hängt mit der Urheberschaft neuer Ideen und ihrer Kritik zusammen. Die Stellung eines Wissenschaftlers in der Gesellschaft, seine Finanzierung sowie die Bereitschaft der Gesellschaft, die Kosten aufwendiger Experimente zu tragen oder bestimmte Lehrmeinungen als Basis weitreichender Entscheidungen zu nehmen bilden ein komplexes ökonomisches und machtpolitisches System, in welchem die Frage der Herkunft einer Idee (,,intellektuelles Eigentum“) besondere Bedeutung hat. Diese Bedeutung bleibt auch dann erhalten, wenn man den traditionellen Begriff des exklusiven ökonomischen Eigentums an Ideen verändert, wie etwa in manchen Open Science, Open Source oder Creative Commons Kreisen.

Aus diesen Beobachtungen ergeben sich die Anforderungen an das Kollaborations- und Kommunikationssystem der Wissenschaft, oder in anderen Worten, den Publikationsprozess: 
1. Dokumentation: Wissenschaftliche Ergebnisse sollen schriftlich festgehalten werden.

2. Kommunikation: Wissenschaftliche Ergebnisse sollen möglichst weit und frühzeitig verbreitet werden und ebenso weithin zugängliche Rückmeldungen, Kritiken und Bewertungen erlauben.

3. Reputation: Ideen und Resultate sollen nachvollziehbar mit ihren Erstautoren verbunden sein. Wissenschaftliche Leistung soll gesellschaftlich und ökonomisch abrechenbar bleiben, um Karrieren und Spezialisierungen zu ermöglichen. Die Gesellschaft hofft, dass Personen, die mehrfach erfolgreich Ideen geschaffen oder bewertet haben, auch in Zukunft erfolgreich sein werden und daher weiter mit Verantwortung betraut werden können.

4. Moderation: Die Bewertung der Ergebnisse soll als Filter und Aufmerksamkeitsmoderator dienen, damit die entstehende Flut an Gedanken und Vorschlägen rezipierbar bleibt, eine Kategorisierung soll spätere Suchvorgänge erleichtern. Ein wichtiger Nebeneffekt der Moderation ist auch die Qualitätssteigerung, da Begutachtung im Idealfall wertvolle Hinweise zur Optimierung des Textes liefert.

Bis Ende des 20. Jahrhunderts gab es noch einen weiteren Faktor, der das wissenschaftliche Verlagswesen dominiert hat, nämlich die Kosten der Übertragung eines Manuskripts aus handschriftlicher oder schlechter maschinschriftlicher Form in gesetzte Druckform, schließlich die Kosten des Druckvorgangs und der Aufwand zur weltweiten Verteilung gedruckter Exemplare. Da diese Kosten durchaus beträchtlich waren, musste auch abgesehen von Reputation und Moderation, bereits aus ökonomischen Motiven heraus eine Vorbegutachtung stattfinden. Wissenschaftsverlage organisierten diese Vorbegutachtung und übernahmen das unternehmerische Risiko, das sich aus der (kostspieligen) Publikation von Ideen ergab, die sich wissenschaftlich möglicherweise nicht durchsetzen konnten und daher auch nicht entsprechend nachgefragt wurden.

Mit der Digitalisierung fertigen nun die meisten Autoren ihre Manuskripte in der endgültigen Form selber an, Fertigung und Verteilung sind fast kostenlos geworden (siehe Infobox 1). Dadurch fällt das unternehmerische Risiko und damit das bisherige Geschäftsmodell der Wissenschaftsverlage weg und die akademische Gepflogenheit des Peer-Reviewing lässt sich nicht mehr als Vorab-Kontrolle mit Hinblick auf Publikationskosten rechtfertigen. Die Begutachtung von Arbeiten behält zwar weiterhin ihre Bedeutung in Bezug auf Reputation und Moderation, wird sich aber ebenso verändern. Insbesondere vermuten wir eine Verschiebung von Bewertungen, die aufgrund einer formalen Gutachterrolle erfolgen, hin zu Bewertungen, die aufgrund originärer Befassung mit der Publikation erfolgen und dabei meist Teil eigener aktiver Forschung sind.

\section{Blogchain als wissenschaftliche Publikationsform}

In diesem Abschnitt stellen wir die „Blogchain“ als neue wissenschaftliche Publikationsform vor. Die Bezeichnung versteht sich als Wortspiel zur besseren Vermarktung und als Hinweis auf Technologie und Intention. Zentrale Wortbestandteile sind 
„Blog“ und „Blockchain“. Mit dem Blog teilt unser Vorschlag das Konzept der offenen, selbstverantworteten, dezentralen und kommentierfähigen Publikationsform, die Blockchain wird zur Absicherung gegen Manipulation durch die Systembetreiber und zur zuverlässigen Verrechnung von Reputation genutzt. Dabei nutzen wir neben der Grundform der Blockchain auch weiterentwickelte Formen mit Smart Contracts, deren technische Möglichkeiten wir hier voraussetzen und dazu auf die einschlägige Literatur verweisen: (Nakamoto 2009), (Buterin 2018), (Wood 2018).

Grundsätzlich ist unser Vorschlag als Metamodell oder Template gedacht. Es umfasst nicht den einen durchgeplanten Geschäftsprozess. Reputation wird nicht auf vorbestimmte Weise auf, beispielsweise, Autoren-Token und Gutachter-Token abgebildet, vielleicht sogar noch mit festgelegtem Wechselkurs. Wir sehen auch keine neuen, spezifischen Metriken, Indizes oder Alt-Metriken vor. Wir stellen jedoch ein Template vor, auf dem viele neue Publikationsformen aufsetzen können. Diese ermöglichen einen Markt neuer Publikationsinstrumente, aus denen sich dann in gegenseitiger Konkurrenz gerade jene Parameter und Gewichte ausbilden, zu deren Festlegung heute die Erfahrung noch fehlt.

Die wesentlichste Änderung betrifft den zeitlichen Ablauf in der Begutachtung und Versionierung einer Arbeit: Im Peer Reviewing wird die Vorbegutachtung zur Reduktion des Kostenrisikos durch eine Nachbegutachtung zur Verrechnung von Reputation ersetzt. Dieser Vorschlag greift tief in das Selbstverständnis des wissenschaftlichen Publikationsprozesses ein und muss auch angesichts der aktuellen Debatte über unseriöse Veröffentlichungen mit Augenmaß umgesetzt werden. Wir glauben, dass die veränderte ökonomische Situation allein schon einen hohen Veränderungsdruck in dieser Richtung erzeugen wird. Ein Dokument wird, nach knapper Vorbegutachtung, welche nur die Funktion einer Art Spam-Schutz übernimmt, in der „Blogchain“ veröffentlicht und dort mit dem Tag „draft“ versehen. In weiteren Tags und Schlüsselwörtern deutet der Autor den Qualitätsanspruch an, den er mit dem Dokument in der vorliegenden Form geltend machen möchte (etwa: Idee, studentische Arbeit, Promotion, A-Journal Fachbeitrag) und die Art seines Beitrags (etwa: Übersicht, didaktische Ausarbeitung, Kommentar, originärer Beitrag). Bewertungen der Arbeit erfolgen nach ihrer Veröffentlichung. Sie können sich aus offiziellen Begutachtungen ergeben, welche die berufenen Gutachter einer Zeitschrift zu diesem Beitrag abgeben, aus Bewertungen von Prüfern in Master- und Promotionsverfahren, aus Kommentaren von Lesern, aus Zitierungen der Arbeit in anderen Arbeiten oder nur aus Lesezugriffen. Für letztere stelle man sich vor, dass die Dokumente in Vollversion nur über das „Blogchain“-Portal zugänglich sind und alle Lesezugriffe gezählt werden. Ein Dokument erwirbt sich also nach seiner Veröffentlichung zunehmend einen höheren Qualitätsausweis durch die Rückmeldungen der Gutachter und Leser. Zugleich kann der Autor sein Dokument überarbeiten, Rückmeldungen aufgreifen und neue Versionen in die „Blogchain“ einstellen. Die „Blogchain“ verwaltet dabei die Dokumente, Rückmeldungen und Kommentare und sichert sie gegen Manipulationen ab. Autoren, Gutachter und Leser können dabei nach Authentisierung namentlich auftreten, aber auch unter wechselnden Pseudonymen agieren. Die einzelnen Akte der Publikation, Begutachtung, Kommentierung, Überarbeitung von Manuskripten usw. werden dabei mit dem Namen bzw. dem jeweils benutzten Pseudonym auf der Blockchain verbunden. Ein Pseudonym ist dabei ein Paar 
aus einem privaten und einem öffentlichen Schlüssel eines asymmetrischen Krypto-Systems. Eigentümer eines Pseudonyms ist, wer die Verfügungsgewalt über den privaten Schlüssel nachweisen kann. Im Ergebnis kann jeder Teilnehmer am System alle Interaktionen mit dem System nachweisen und beispielsweise die Autorenschaft eines bestimmten Papiers oder Gutachtens nachweisen. Das auf diesem Metamodell aufsetzende konkrete digitale Journal kann damit weiterhin seinen etablierten Ablauf beibehalten und die gewünschten Phasen der doppelt-blinden Begutachtung sowie eine schrittweise Aufhebung der Anonymität. Der Verlag, der sich seine Rolle als Buchhalter der Reputation teuer bezahlen lässt, wird in dieser Rolle überflüssig, da die Blockchain die Beweissicherung übernimmt.

In dieser neuen Publikationsform können auch andere Prinzipien des Verlagswesens umgestaltet werden. Die Bedeutung einer Zeitschrift lag bisher in ihrer Rolle als Auslieferungs-, Abrechnungs- und Copyright-Einheit mit einheitlichem Qualitätsstandard. Der informierten Leserschaft ist klar, was sie bei einem Exemplar von „Theoretical Computer Science“ oder „Nature“ zu erwarten hat. Zugleich war das Heft auch Grundlage von Urheberrecht und damit Abrechnung: Ein Beitrag erschien eben nur in „Nature“, wollte man ihn lesen, so musste man diesen Beitrag kaufen oder ein Abonnement der Zeitschrift vorhalten. Mit dem Wegfall der physikalischen Auslieferung werden auch die Abrechnungs- und Urheberrechts-Aspekte obsolet. Die einzige verbleibende Rolle einer Zeitschrift für den Leser ist damit jene eines Aufmerksamkeitsmoderators, der über die erwarteten Inhalte, Formen und Qualitätsstandards wirkt: Man liest „Nature“, weil man in der Zielgruppe dieser Marke ist, man publiziert in „Nature“, weil man diese Zielgruppe erreichen will und seine Fähigkeiten als Autor dieser Qualitätsklasse dokumentieren möchte. Weil die bisherigen Gründe für Exklusivität, insbesondere die ökonomischen und urheberrechtlichen Aspekte, wegfallen, gibt es auch keine Notwendigkeit mehr, dass jeder Beitrag in höchstens einer Zeitschrift abgedruckt wird - von Wiederabdrucken besonders relevanter Artikel einmal abgesehen. Im Gegenteil: Zeitschriften verstehen sich gleichsam als Leitseite für bestimmte Inhalte und Qualitäten und können, wie Internet Link-Listen, einen besonders guten Artikel aufnehmen. Damit kann eine Publikation in mehreren Journalen erscheinen und dadurch besondere Attraktivität und Qualität dokumentieren.

Ein auf dieser Basis organisiertes Publikationswesen generiert zunächst keine Einkünfte und verfügt damit für die geringen, aber gleichwohl vorhandenen Kosten über keinen Deckungsbeitrag. Folgt man unserer obigen Argumentation über digitale Disruption, so ist unserem Vorschlag damit dasselbe Nischendasein bestimmt, wie den dezentralen sozialen Netzwerken. Um dieses zu vermeiden und die notwendigen Geldmittel für Organisation und Weiterentwicklung bereit zu stellen, aber auch um weiteren Mehrwert zu generieren, ist für jeden Teilnehmer ein kleiner Mitgliedsbeitrag an der „Blogchain“ vorgesehen. Für diesen erwerben sich die Nutzer das Recht zur Teilnahme an der Kooperationsplattform und eine Stellung als StakeHolder. Anders als bei Wissenschaftsportalen wie https://www.researchgate.net oder https://www.academia.edu sind ihre Profile und ihre Aufmerksamkeit nicht mehr das Produkt, das von den Portalen nach einem unklaren Geschäftsmodell vermarktet wird, sondern sie sind die Auftraggeber eines von ihnen genutzten Dienstes. Aus dem langfristigen Kommunikationsmodell (Einreichung - Begutachtung - Pu- 
blikation - Kommentierung) wird damit ein flexibles Modell mit geringer Latenz, das sogar tägliche Rückmeldungen, Begutachtungen und Revisionen gestattet. Wissenschaftsblogs oder wissenschaftliche Q \& A Sites ${ }^{6}$ nutzen dieses Format schon lange, die dort eingespeisten Ideen gehen jedoch der Reputationsbuchhaltung einer akademischen Karriere verloren.

\section{Schritte zur praktischen Umsetzung}

Für die technische Umsetzung werden drei wesentliche Bausteine benötigt: (1) Ressourcen für die dauerhafte und zuverlässige Speicherung der Beiträge, (2) Benutzerschnittstellen und Portale zur Abwicklung der Speicherung in Upload und Download sowie zur Suche in erfolgten Publikationen und schließlich (3) eine Instanz zur Manipulationssicherung durch die jeweiligen Systembetreiber und zur authentischen Verbindung aller Beiträge, Gutachten und Kommentare mit den ausführenden pseudonymen oder namentlich identifizierten Benutzern auf Basis einer Blockchain.

Die Speicher und Benutzerschnittstellen können von Universitätsbibliotheken bereitgestellt werden, so wie es heute für die digitalen Formen klassischer Medien bereits vielfach und erfolgreich geschieht. Diese Systeme können durch Knoten unter der Verantwortung einzelner Wissenschaftler oder Institute erweitert werden. Eine Auslagerung an kommerzielle Dienstleister, welche für die entsprechenden Aufgaben dann nach marktkonformen Kriterien entlohnt werden, ist ebenso denkbar. Auch in der Bitcoin- oder in der Ethereum-Blockchain arbeiten Mining Pools, Unternehmen und individuelle Miner zu gleichen und marktüblichen Bedingungen an der Erweiterung der Blockkette mit.

Zur Absicherung gegen Manipulation durch die einzelnen Betreiber wird die Blockchain-Technologie eingesetzt. Dazu haben wir in (Grabinsky 2018) verschiedene architekturelle Möglichkeiten untersucht. So können die Publikationen und Kommentare etwa vollständig in der Ethereum-Blockchain abgelegt werden; die Speicheranforderungen der entsprechenden, in Solidity geschriebenen Smart Contracts, sind jedoch so hoch, dass die Blockchain Fees die derzeit üblichen Open Access Seitenkosten übersteigen dürften. Sinnvoller erscheint daher die Speicherung in einem separaten Dienst, der gegen Manipulationen durch die Ablage von kryptographischen Hash-Werten auf der Blockchain abgesichert wird. Diesen Weg gehen etwa auch die Blockchain-basierten Speicherdienste des Interplanetary File Systems (IPFS, siehe https://ipfs.io). Es ist aber ebenso denkbar, dass für die Realisierung eines verteilten Speicherdienstes ein eigener Kryptotoken verwendet wird, der zugleich als Währung zur Bezahlung der Mitwirkenden an diesem Dienst genutzt werden kann. Diesen Weg beschreitet etwa das Startup Filecoin (siehe https:// filecoin.io).

\footnotetext{
${ }^{6}$ Etwa die Familie der Stack Exchange Websites.
} 
Als Proof-of-Concept für unsere Idee hat (Buschendorf 2018) mit ihrem System FakeChair ${ }^{7}$ eine Software zur Verwaltung von Konferenzbeiträgen nach dem Vorbild von EasyChair implementiert. Der Funktionsumfang ist minimal: Es können Tagungen angelegt werden, Autoren und Gutachter hinzugefügt werden; Autoren können Papiere hochladen und Gutachter können Bewertungen abgeben; publizierte Beiträge können von jedem gelesen werden. Kommentare und andere Formulareingaben werden als Json-Objekte erfasst und über die web3 JavaScript BlockchainAPI und das MetaMask Browser-Plugin an die Ethereum-Blockchain weitergereicht. Größere Dateien werden im IPFS gespeichert und durch Hash-Referenzen an den entsprechenden Smart Contract auf der Blockchain angebunden.

\section{Bewertung, vergleichbare Ansätze und Ausblick}

Wir haben ein Metamodell als alternatives Vorgehen für wissenschaftliches Publizieren vorgestellt. Als Mehrwert ergibt sich daraus zunächst eine deutliche Kostensenkung der derzeit hochpreislichen Zeitschriftenversorgung in den Wissenschaften. Wir hoffen, dass auf Basis unserer Vision auch jenseits der Diskussionen über Preiserhöhungen, Boykotte ${ }^{8}$ und weitere Aspekte ${ }^{9}$ tragfähige Formen der Literaturversorgung von Wissenschaftlern für Wissenschaftler aufgebaut werden können.

Neben dem ökonomischen Mehrwert erlaubt unser Modell viele weitere Vorteile. Die Bindung von Ideen an ihre Autoren erlaubt eine frühzeitige Publikation und Diskussion ohne Gefahr des Verlusts der Urheberschaft. Dynamische Weiterentwicklung der Dokumente nach und während der Begutachtung flexibilisiert das Reviewing und gewährleistet die Verrechnung wissenschaftlicher Reputation. Die Natur unseres Vorschlags als Metamodell ermöglicht die exakte Beibehaltung bisherige traditioneller Abläufe, macht aber zentrale Instanzen überflüssig. Diese Eigenschaft erscheint uns angesichts der eher konservativen Sichtweise in den Wissenschaften, die Bewährtes bewahren möchte, sinnvoll. Sie gestattet aber auch den schrittweisen Umbruch bis hin zu radikalen Neuerungen. Vorstellbar wären etwa die kontinuierliche öffentliche Dokumentation einer Forschungsidee und ihre Begleitung durch Kommentare und Gutachten, die von den ersten Anfängen schrittweise zu ersten Ergebnissen bis hin zur fertigen Doktorarbeit führt und deren Beiträge als lebenslaufwirksame Publikationen und schließlich als angenommene Doktorarbeit zählen, da parallel zur Weiterentwicklung der Arbeit auch entsprechende Begutachtungen eingespeist werden. Der Grad an öffentlicher Einsehbarkeit und Anonymität kann dabei jeweils gesteuert werden. Wesentlich für unseren Vorschlag ist die nach-

\footnotetext{
7 Source Code verfügbar auf dem Github Repository FabiolaBusch/fakechair. Der Demonstrator unter der URL http://fakechair.fabiolabuschendorf.de/ setzt die Installation der Blockchain Browser-Extension MetaMask voraus.

8 Siehe etwa: The Cost of Knowledge, http://thecostofknowledge.com.

9 Siehe etwa: Jon Tennant: Elsevier are corrupting open science in Europe. The Guardian, 29. Juni 2018. https://www.theguardian.com/science/political-science/2018/jun/29/elsevier-are-corrupting-openscience-in-europe.
} 
vollziehbare Authentisierung der einzelnen Schritte und ihre Absicherung gegen Manipulation.

Es bestehen ähnlich Ansätze und Überlegungen im Umfeld von Blockchain-basierten Initial Coin Offerings sowie bei wissenschaftlichen Fachgesellschaften. So beschreibt (Spearpoint 2017) die Idee des R-coin, mit dem sich die Beteiligung an Gutachten und Arbeiten messen lässt und der die Rolle einer neuen Altmetric übernehmen kann. Das Modell erscheint uns zu eingeschränkt, da es die Monetarisierung einzelner Aktivitäten in den Vordergrund stellt und daher ein „Gaming“ des Systems erlaubt, wie es bei traditionellen Forschungsmetriken und Zitationskartellen weithin bekannt ist. Das Portal Steemit verfolgt ähnliche Ziele, schiebt aber die monetäre Seite noch stärker in den Vordergrund; es dürfte sich daher stärker für kommerziell orientierte Publikationen eignen, da es auch auf käuflich erwerbbare Rechte in der Plattform abstellt.

Für unsere Idee hat Grabinsky (2018) die grundsätzlichen theoretischen Möglichkeiten zur Realisierung aufzeigt und FakeChair von Buschendorf (2018) die praktische Machbarkeit längs einer der vielen möglichen Implementierungsstrategien nachgewiesen. Wir wollen nun praktische Erfahrungen sammeln und insbesondere die Frage untersuchen, wie das soziale System aus Autoren und Gutachtern auf unser Konzept reagiert. Neben einigen Erweiterungen der Implementierung, speziell im Bereich der Benutzerführung, müssen dazu noch Unterstützungs-Strukturen (backoffice) implementiert werden.

Die wesentliche Herausforderung besteht in der Reaktion der unterschiedlichen Wissenschafts- und Begutachtungskulturen der verschiedenen Fachdisziplinen. Unser Vorschlag umfasst ganz bewusst nur ein Metamodell. Damit fehlen ihm wichtige Elemente eines konkreten Publikationsorgans, das seinen Kunden detailliert ausgearbeitete Abläufe anbieten muss und die in der Disziplin etablierten Gebräuche reflektieren sollte. Die Bandbreite reicht von der Anzahl und Ausführlichkeit der Begutachtungen bis hin zum „Wechselkurs“ zwischen den „Reputationspunkten“ für die Autoren, Gutachter und Kommentatoren. Der nächste Schritt muss also aus einem aufwendigen Experimentieren bestehen, in dem zunächst auch Raum für ein Scheitern und Chance für ein kontrolliertes Nachjustieren der spezifischen Parameter besteht. Wir hoffen, dass wir dazu Gelegenheit und Unterstützung finden.

Danksagung Die Autoren bekamen aus der anonymen Begutachtung hilfreiche und wichtige Kommentare zur Verbesserung des Artikels. Wir bedanken uns dafür ganz herzlich!

Open Access Dieser Artikel wird unter der Creative Commons Namensnennung 4.0 International Lizenz (http://creativecommons.org/licenses/by/4.0/deed.de) veröffentlicht, welche die Nutzung, Vervielfältigung, Bearbeitung, Verbreitung und Wiedergabe in jeglichem Medium und Format erlaubt, sofern Sie den/die ursprünglichen Autor(en) und die Quelle ordnungsgemäß nennen, einen Link zur Creative Commons Lizenz beifügen und angeben, ob Änderungen vorgenommen wurden. 


\section{Literatur}

Brewer E (2000) Towards robust distributed systems. ACM PODC 2000. (Keynote http://www.eecs. berkeley.edu/ brewer/cs262b-2004/PODC-keynote.pdf)

Buschendorf F (2018) Implementation of a peer-reviewing platform on the Blockchain (Universität Göttingen, Bachelor Arbeit, Juni 2018)

Buterin V (2018) Ethereum whitepaper: a next-generation smart contract and decentralized application platform (Github.com/ethereum)

Gilbert S, Lynch N (2002) Brewer's conjecture and the feasibility of consistent, available, partition-tolerant web services. ACM SIGACT News 33(2):51-59

Grabinsky M (2018) Konzeption einer dezentralisierten App mittels distributed ledger Technologien zur Veröffentlichung und Verwaltung von wissenschaftlichen Ergebnissen (Universität Rostock, MasterArbeit, Oktober 2018)

Hartig K (2017) Replizierbarkeit von Forschungsergebnissen. Eine Stellungnahme der Deutschen Forschungsgemeinschaft. DFG, Bonn

Johnston L (2012) A "library of congress" worth of data: it's all in how you define it. https://blogs.loc. gov/thesignal/2012/04/a-library-of-congress-worth-of-data-its-all-in-how-you-define-it/. Zugegriffen: 18. Juli 2018

Nakamoto S (2009) Bitcoin: a peer-to-peer electronic cash system. Working paper. http://bitcoin.org/ bitcoin.pdf

Shen X, Yu H, Buford J, Akon M (Hrsg) (2009) Handbook of peer-to-peer networking. Springer, Heidelberg, Berlin, New York

Spearpoint M (2017) A proposed currency system for academic peer review payments using the Blockchain technology. Publications 5:19

Stölting E, Schimank U (Hrsg) (2001) Die Krise der Universitäten. Springer, Wiesbaden

Wood G (2018) Ethereum yellowpaper: Ethereum: a secure decentralized generalized transaction ledger byzantium version. Ethereum.github.io, June 2018 